\title{
Enzyme-free monitoring of glucose utilization in stimulated macrophages using carbon nanotube-decorated electrochemical sensor
}

\author{
Sasya Madhurantakam ${ }^{1,2} \cdot$ Jayanth Babu Karnam $^{3} \cdot$ John Bosco Balaguru Rayappan $^{1,3}$. \\ Uma Maheswari Krishnan ${ }^{1,2}$
}

Received: 21 August 2017/ Accepted: 7 October 2017/Published online: 20 October 2017

(c) The Author(s) 2017. This article is an open access publication

\begin{abstract}
Carbon nanotubes (CNTs) have been extensively explored for a diverse range of applications due to their unique electrical and mechanical properties. CNT-incorporated electrochemical sensors have exhibited enhanced sensitivity towards the analyte molecule due to the excellent electron transfer properties of CNTs. In addition, CNTs possess a large surface area-to-volume ratio that favours the adhesion of analyte molecules as well as enhances the electroactive area. Most of the electrochemical sensors have employed CNTs as a nano-interface to promote electron transfer and as an immobilization matrix for enzymes. The present work explores the potential of CNTs to serve as a catalytic interface for the enzymeless quantification of glucose. The figure of merits for the enzymeless sensor was comparable to the performance of several enzyme-based sensors reported in literature. The developed sensor was successfully employed to determine the glucose utilization of unstimulated and stimulated macrophages. The significant difference in the glucose utilization levels in activated macrophages and quiescent cells observed in the present investigation opens up the
\end{abstract}

Uma Maheswari Krishnan

umakrishnan@sastra.edu

1 Centre for Nanotechnology \& Advanced Biomaterials (CeNTAB), SASTRA University, Thanjavur, Tamil Nadu 613 401, India

2 School of Chemical \& Biotechnology, SASTRA University, Thanjavur 613 401, India

3 School of Electrical and Electronics Engineering, SASTRA University, Thanjavur 613 401, India possibilities of new avenues for effective medical diagnosis of inflammatory disorders.

Keywords Multi-walled carbon nanotubes · Enzymeless detection - Electrochemical biosensor - Glucose . Macrophages

\section{Introduction}

Glucose metabolism is a central cellular function and elevated glucose levels beyond $5.5 \mathrm{mM}$ in blood and low or high uptake of glucose by the cell results in several disorders. Proper monitoring of glucose levels will help to reduce the risk of diabetes, atherosclerosis, diabetic retinopathy, kidney failure, nerve degeneration, etc. (Zhu et al. 2012). Elevated glucose levels have become a major clinical parameter for the diagnosis of diabetes and it is also used to decide the dosage of insulin to patients. Glucose has also been identified as a critical component in the pro-inflammatory response of macrophages (Freemerman et al. 2014). Macrophages are key components of our cellular immunity and play a major role in the deposition of plaques in arteries leading to atherosclerosis. Macrophages react with low-density lipoproteins and create foam cells, which in turn disintegrate to form crystal-like cholesterol structures. The cholesterol crystals activate new macrophages causing them to release inflammatory cytokines and amplify inflammation (Maltby et al. 2009; Numata et al. 2016). Hence, quantification of glucose levels in macrophages in normal and stimulated conditions can be an ideal system to predict the risk of macrophage-assisted atherosclerosis. Furthermore, it can also be an indispensable clinical parameter for the diagnosis of diabetes and atherosclerosis risk and also help in the design of 
appropriate treatment regimen. Such strategy has not been explored thoroughly in the realm of healthcare sensors and has been attempted for the first time in the present work.

In the context of rapid diagnosis, electrochemical sensors have gained popularity due to their low cost, easy preparation, sensitivity, rapid response, and simple operation that enables self monitoring by an individual (Wang et al. 2008b; Harper and Anderson 2010; Kimmel et al. 2013). The sensing element widely employed in electrochemical glucose sensors is glucose oxidase enzyme immobilized on the electrode surface. Most of the commercially available glucometers are enzyme-based because of the high sensitivity and good selectivity of the enzyme towards the analyte (Wang et al. 2011; Chen et al. 2012; Zhang et al. 2014). The first-generation glucose sensors monitored oxygen content at the electrode, while the second-generation sensors used a redox mediator to measure the signal. The third-generation sensors utilized direct electron transfer at the electrode surface to measure the current. However, the electron transfer between the immobilized enzyme and the electrode surface was slow. This prompted the development of nano-structured interfaces that facilitated rapid electron transfer. However, enzymatic sensors are limited (Wang et al. 2008a) by their instability due to changes in temperature and $\mathrm{pH}$ (Park et al. 2006; Wang et al. 2013; Li et al. 2015). This has led to the development of the fourth generation of electrochemical sensors that are enzyme-free. The catalysis is brought about by nanodimensional particles at the electrode surface. The nano-interface possesses high surface area-to-volume ratio, fast and direct electron transfer, desirable electrical and mechanical properties, as well as the capability of adsorbing biomolecules (Dong and Chen 2002; Asefa et al. 2009). A majority of non-enzymatic sensors have employed metallic nanoparticles such as platinum (Yuan et al. 2005; Cao et al. 2007), gold (Ma et al. 2009), nickel (Salimi et al. 2004; Sue et al. 2008), copper (Sattayasamitsathit et al. 2009; Chen et al. 2012), bimetallic systems such as $\mathrm{Pt}-\mathrm{Pb}$ and metal oxides such as $\mathrm{CuO}$ (Reitz et al. 2008; Wang et al. 2010), $\mathrm{ZnO}$ (Lei et al. 2010), $\mathrm{TiO}_{2}$ (Lo et al. 2008) alone or in combination with carbon nanostructures such as single-walled ( $\mathrm{Su}$ et al. 2010) and multi-walled carbon nanotubes (Chen et al. 2008), graphene (Zhu et al. 2010), etc. The use of carbon nanotubes along with metallic nanoparticles have been found to enhance the electron transfer, while the catalysis is performed by the metallic centres. However, the presence of chloride in the electrolyte has been found to interfere with the catalytic ability of many metallic nanoparticles. Hence, the possibility of exploiting the independent electrocatalytic nature of carbon nanotubes for the measurement of glucose has been attempted in this study. Only few reports are available on the electrocatalytic nature of pristine CNTs towards sensing applications. These sensors were investigated in simulated samples and no real-time analysis was performed in the earlier studies (Ye et al. 2004). In the present study, a mildly alkaline electrolyte is employed instead of the highly alkaline systems reported earlier to ensure that there are no adverse effects on the biological sample being investigated. Macrophages were stimulated using lipopolysaccharides to monitor the glucose utilization by the cells. Glucose utilization was measured in macrophages both in unstimulated and stimulated conditions in an effort to understand differences between these two conditions that can be used for possible detection of inflammatory conditions.

\section{Materials and methods}

\section{Materials}

D-Glucose (Hi-media), nafion (M/s Sigma-Aldrich Ltd), and multi-walled carbon nanotubes (CNTs) (Nanomor, USA) were used in the study. Lipopolysaccharide (LPS) was procured from (M/s Sigma-Aldrich Ltd). Sodium hydroxide, potassium dihydrogen phosphate, dipotassium hydrogen phosphate, acetic acid, ascorbic acid, citric acid, and uric acid were purchased from Merck, India. Glassy carbon electrode (GCE), $\mathrm{KCl}$ saturated $\mathrm{Ag} / \mathrm{AgCl}$ reference electrode, and platinum wire counter electrodes were purchased from CH Instruments, Inc., USA, and used for the electrochemical measurements. Deionized water was used to prepare all solutions.

\section{Modification of working electrode}

The glassy carbon electrode was cleaned as mentioned elsewhere (Thiagarajan et al. 2016). Carbon nanotubes (CNT) were dispersed in $0.05 \%$ of nafion solution and sonicated for $1 \mathrm{~h}$. Then, $3 \mu \mathrm{L}$ of this solution was dropcasted on the surface of the glassy carbon electrode and dried for $1 \mathrm{~h}$. This electrode was denoted as CNT/GCE and was used as the working electrode for electrochemical measurements.

\section{Characterization}

The morphology of carbon nanotubes was observed using field-emission scanning electron microscopy (FE-SEM, JSM 6701F, JEOL, Japan). The sample was sputter-coated with a thin layer of gold and imaged at an accelerating voltage of $3 \mathrm{kV}$. The quality and purity of the carbon nanotubes were evaluated using Raman spectroscopy (LabRam HR800, Horiba Scientific, Japan). The spectrum 
was acquired between 0 and $2000 \mathrm{~cm}^{-1}$ in a moisture-free environment.

\section{Electrochemical measurements}

The electrochemical analyses were carried out using an electrochemical analyzer (CHI600C, CH Instruments, USA). A three-electrode system was employed for cyclic voltammetry and amperometry recordings. Platinum wire was used as the counter electrode, while $\mathrm{Ag} / \mathrm{AgCl}$ electrode (saturated, $0.1 \mathrm{M} \mathrm{KCl}$ ) was employed as the reference. The CNT/GCE served as the working electrode. Cyclic voltammograms were recorded in phosphate buffered saline medium (PBS, $\mathrm{pH} 7.4$ ) at $298 \mathrm{~K}$ and a scan rate of $0.01 \mathrm{Vs}^{-1}$. The amperometric measurements were performed at time intervals of $100 \mathrm{~s}$ following addition of specific concentration of glucose at an applied potential of $-0.25 \mathrm{~V}$. Each step in the amperometric profile corresponds to the addition of $1 \mathrm{mM}$ glucose.

\section{Cell culture studies}

Mouse macrophage cells (IC-21) were cultured in Dulbecco's Modified Eagles Medium (DMEM) with fetal bovine serum (FBS, 10\%) and penicillin/streptomycin (1\%, Gibco, USA) and incubated in $5 \% \mathrm{CO}_{2}$ at $37{ }^{\circ} \mathrm{C}$. After cells attained confluency, they were trypsinized and centrifuged for $5 \mathrm{~min}$ at $4000 \mathrm{rpm}$. After discarding the supernatant, the cells were suspended in $1 \mathrm{~mL}$ of DMEM. The cells were seeded with different densities $(1,2,5,8$, and $10 \times 10^{3}$ cells) in two sets. One set of cells was maintained in DMEM, while another set of cells was supplemented with LPS containing DMEM. After $24 \mathrm{~h}$, the spent medium was collected and glucose measurements were made using the developed electrochemical sensor.

\section{Results and discussion}

\section{Characterization}

The surface morphology and Raman spectrum of the aspurchased CNTs are shown in Fig. 1.

Figure 1a reveals the presence of a dense network of uniform-sized nanotubes with an average diameter of 40-50 nm. The Raman spectrum of the CNTs shown in Fig. 1b displays a lower intensity $D$ band at $1320 \mathrm{~cm}^{-1}$ and a higher intensity $G$ band at $1573 \mathrm{~cm}^{-1}$. These values are in agreement with earlier reports for MWCNTs (Bokobza and Zhang 2012). The presence of the radial breathing mode (RBM) at $253 \mathrm{~cm}^{-1}$ provides additional support for the presence of MWCNTs.

\section{Electrochemical measurements}

Figure 2 shows the cyclic voltammograms recorded for the bare GCE and CNT-coated GCE working electrode in the presence of $1 \mathrm{mM}$ glucose. An oxidation peak appears at $-0.25 \mathrm{~V}$ in the modified electrode in the presence of glucose. No peak is observed for bare GCE in the presence of glucose. The oxidation peak in the CNT-coated GCE arises due to the conversion of glucose to gluconic acid that is facilitated by the CNTs on the electrode surface.

Earlier reports have indicated that the glucose oxidation can occur in alkaline medium and the electron transfer can be mediated by the presence of carbon nanotubes and remains unaffected by chloride ions in the medium (Wang et al. 2007). While the earlier reports had employed sodium hydroxide containing chloride ions as the electrolyte, the present study has used mildly alkaline phosphate buffered saline ( $\mathrm{pH}$ 7.4) that also contains chloride ions. The appearance of the glucose oxidation peak indicates that the mildly alkaline conditions provided by the phosphate buffered saline is sufficient for the oxidation of glucose to gluconic acid catalyzed by the CNT-modified surface that also captures the resultant electron transfer efficiently. Though the exact mechanism for the electrocatalytic behavior of MWCNTs has not yet been deciphered, it is believed that a combination of defect sites at the cap and the presence of oxygen-containing functional groups contribute to the electrocatalytic effect of MWCNTs ( $\mathrm{Hu}$ and $\mathrm{Hu}$ 2009). The alkaline medium has been reported to activate the surface functionalities in MWCNTs (Ye et al. 2004). Moreover, the surface roughness also contributes in enhancing the sensitivity of the CNT-modified surface towards the analyte. According to the Incipient Hydrous Oxide Adatom Mediator model (IHOAM), the defective sites in the CNTs facilitate the adsorption of the hydroxyl ions that catalyze the oxidation of glucose to gluconic acid (Niu et al. 2016). However, the participation of the trace amounts of metal catalyst used for the preparation of CNTs in the catalytic transformation also cannot be ruled out. The nafion coating retards diffusion of larger molecules, thereby limiting interference. Figure 3 depicts the schematic representation of the reaction occurring at the working electrode.

Figure 4 shows the effect of scan rate on the oxidation peak in the presence of $1 \mathrm{mM}$ of glucose. The peak current shows a progressive increase in the presence of glucose at increasing scan rates. The linear relation between scan rate and current indicates that the oxidation reaction is confined to the surface of the electrode.

The concentration-dependent response of the developed biosensor was determined by increasing the concentrations of glucose. It was observed that the current progressively decreased with an increase in the concentration of glucose 


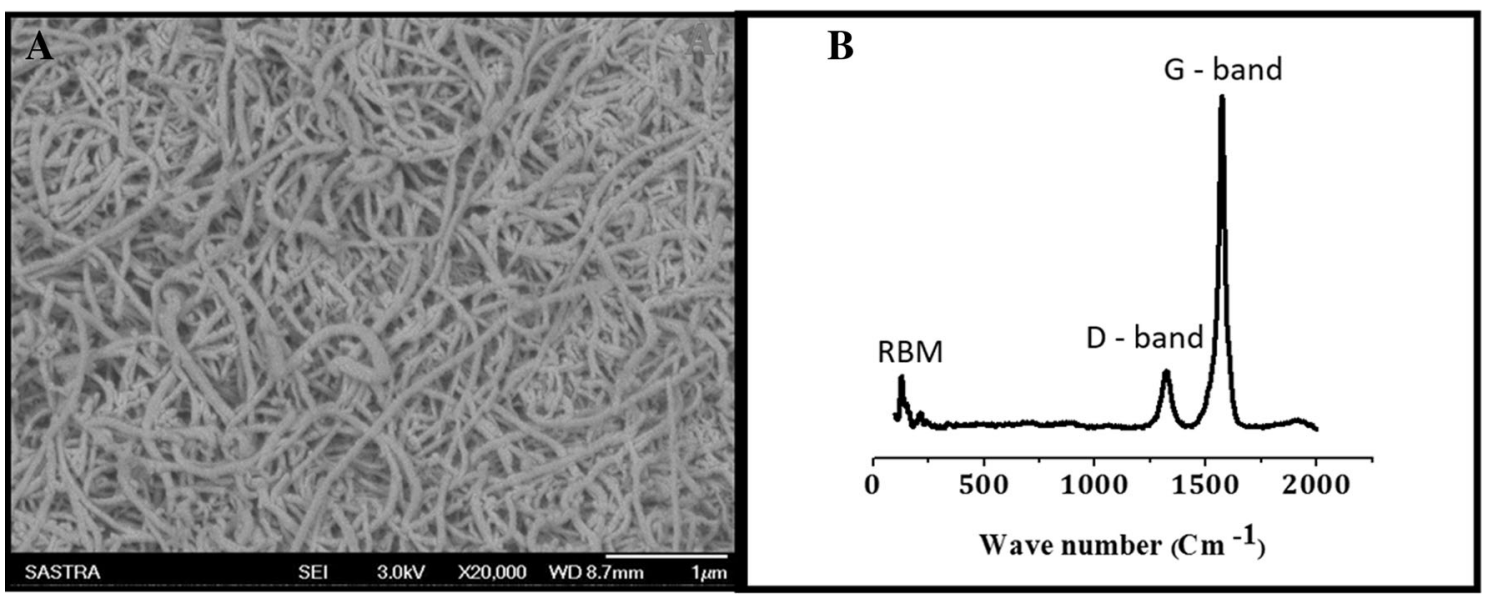

Fig. 1 a Scanning electron micrograph and $\mathbf{b}$ Raman spectrum of carbon nanotubes

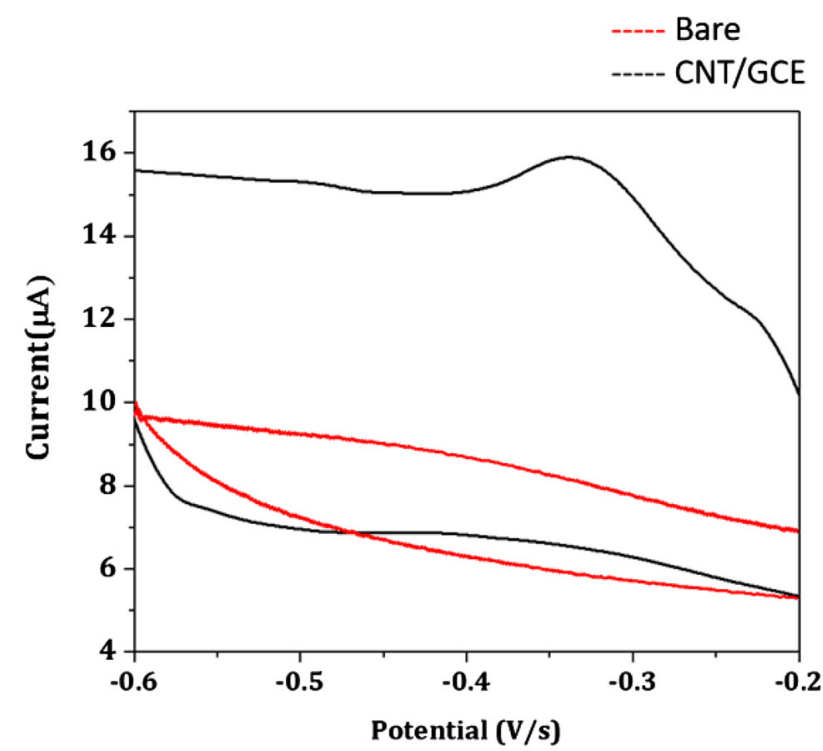

Fig. 2 Cyclic voltammogram of bare GCE and CNT-coated GCE in the presence of $1 \mathrm{mM}$ glucose in PBS ( $\mathrm{pH} 7.4$ )

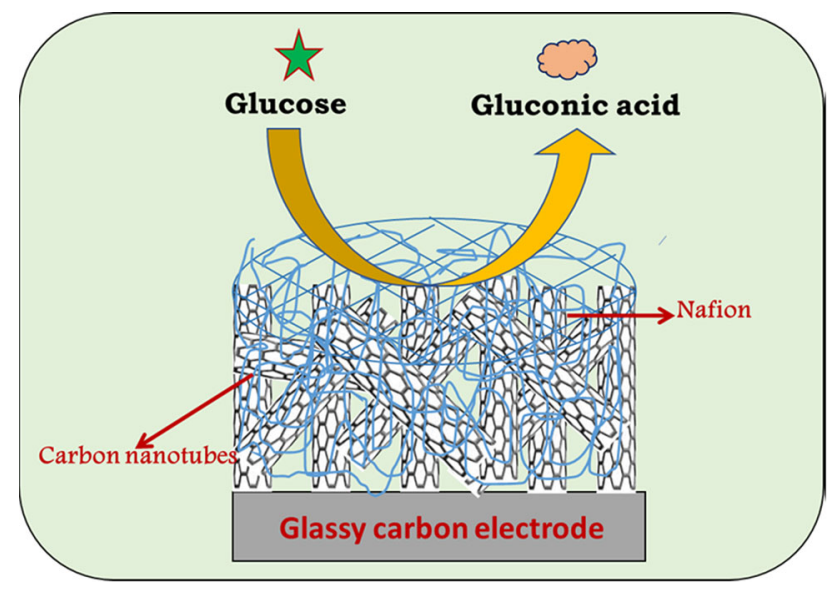

Fig. 3 Schematic representation of reaction mechanism occurring at the working electrode up to $5 \mathrm{mM}$ (Fig. 5). The plot of concentration versus current exhibits a linear trend with a negative slope and linear regression of 0.98 . This clearly indicates that the oxidation reaction is a surface controlled phenomenon at the CNT-coated GCE.

Figure 6a shows the amperometric profile recorded for progressive addition of glucose to the medium at an applied voltage of $-0.32 \mathrm{~V}$. A linear relationship is obtained for the concentration and current that can be used to quantify the amount of glucose present in the sample (Fig. 6b). The decrease in peak current may be attributed to adsorption of gluconic acid on CNT and electrode. The formation of hydrogen bond between carboxylic functiional group and defective site in CNT may leads to the adsorption of gluconic acid on CNT (Dantas et al. 2014). Response time is the time required to achieve stable state of sensor response toward the substrate from the moment of its addition to the electrolyte. The time taken for the sensor to achieve stable current after addition of the sample was found to be $3 \mathrm{~s}$.

The plot of current versus concentration of glucose (Fig. 6b) shows a linear regression of 0.986 and a linear range from 2 to $8 \mathrm{mM}$ of glucose. The various sensing parameters for the enzymeless sensor were calculated and are summarized in Table 1.

It is evident from Table 1 that the sensor exhibits a limit of detection of $1.59 \mathrm{mM}$ and a linear range of $2-8 \mathrm{mM}$. The normal concentration of blood glucose is $4-5.5 \mathrm{mM}$ (Suneesh et al. 2013), which lies in the working range of this enzymeless sensor.

A comparison of the different enzymeless glucose sensors reported in literature is given in Table 2 .

\section{Effect of interferents}

The designed biosensor was tested towards the potential interferents like ascorbic acid, uric acid, citric acid, etc. 

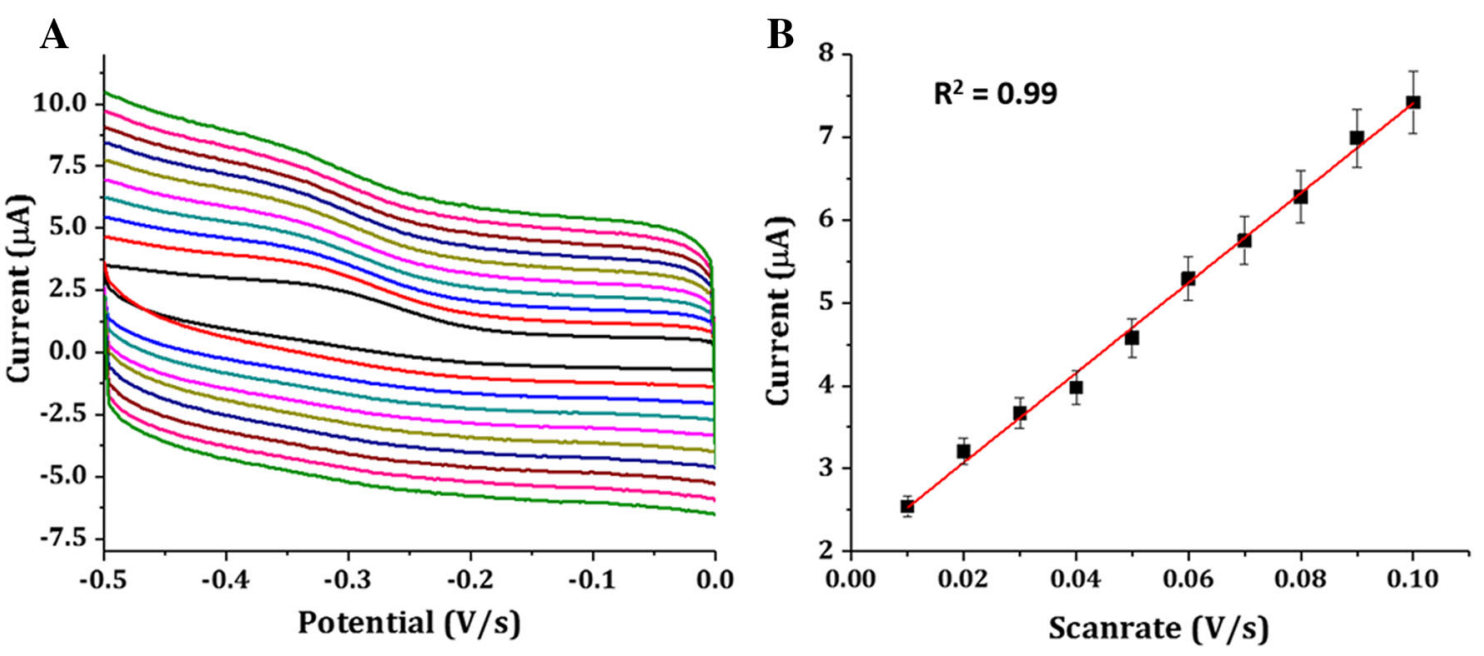

Fig. 4 a Cyclic voltammograms of CNT/GCE by varying the scan rate from 0.01 to $0.1 \mathrm{~V} / \mathrm{s}$ and $\mathbf{b}$ plot of scan rate versus current

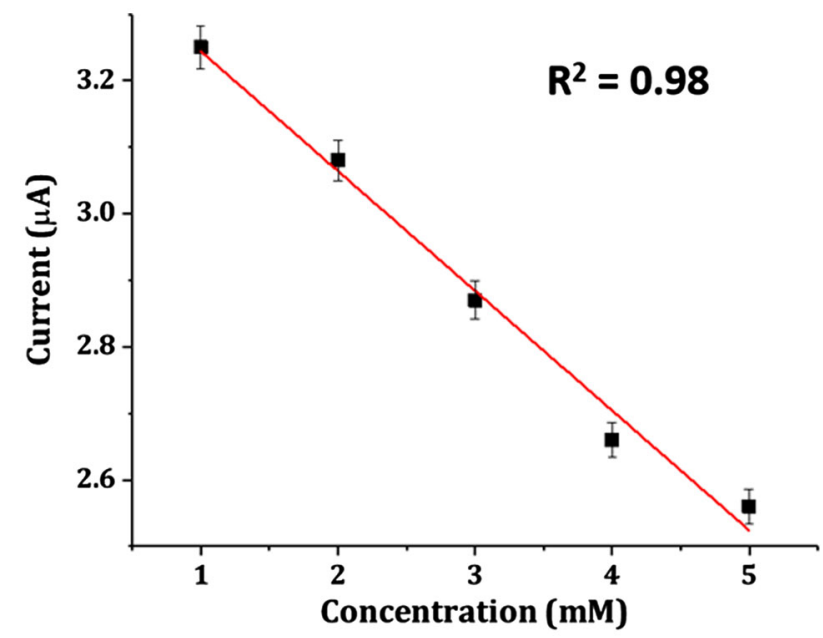

Figure 7 shows the amperometric response of biosensor towards $1 \mathrm{mM}$ glucose and other interferents at $-0.32 \mathrm{~V}$ in PBS. The concentration of each interferent was $1 \mathrm{mM}$. From Fig. 7, it is evident that the sensor responds only to

Table 1 Various sensing parameters of the developed biosensor with their formula and values

\begin{tabular}{lll}
\hline Sensor parameter & Formula & Value \\
\hline Area of the working electrode & $\pi r^{2}$ & $0.12 \mathrm{~cm}^{2}$ \\
Surface coverage $(\Gamma)$ & $Q / n \mathrm{FA}$ & $14.27 \mathrm{nM} / \mathrm{cm}^{2}$ \\
Limit of detection & {$[3.3 \times \mathrm{SD}] /$ slope } & $1.59 \mathrm{mM}$ \\
Limit of quantification & {$[10 \times \mathrm{SD}] / \mathrm{slope}$} & $4.83 \mathrm{mM}$ \\
Theoretical sensitivity & $I_{\max } / K_{\mathrm{m}}$ & 2.06 \\
Practical sensitivity & Slope & 3.15 \\
\hline
\end{tabular}

Fig. 5 Plot of concentration of glucose versus current
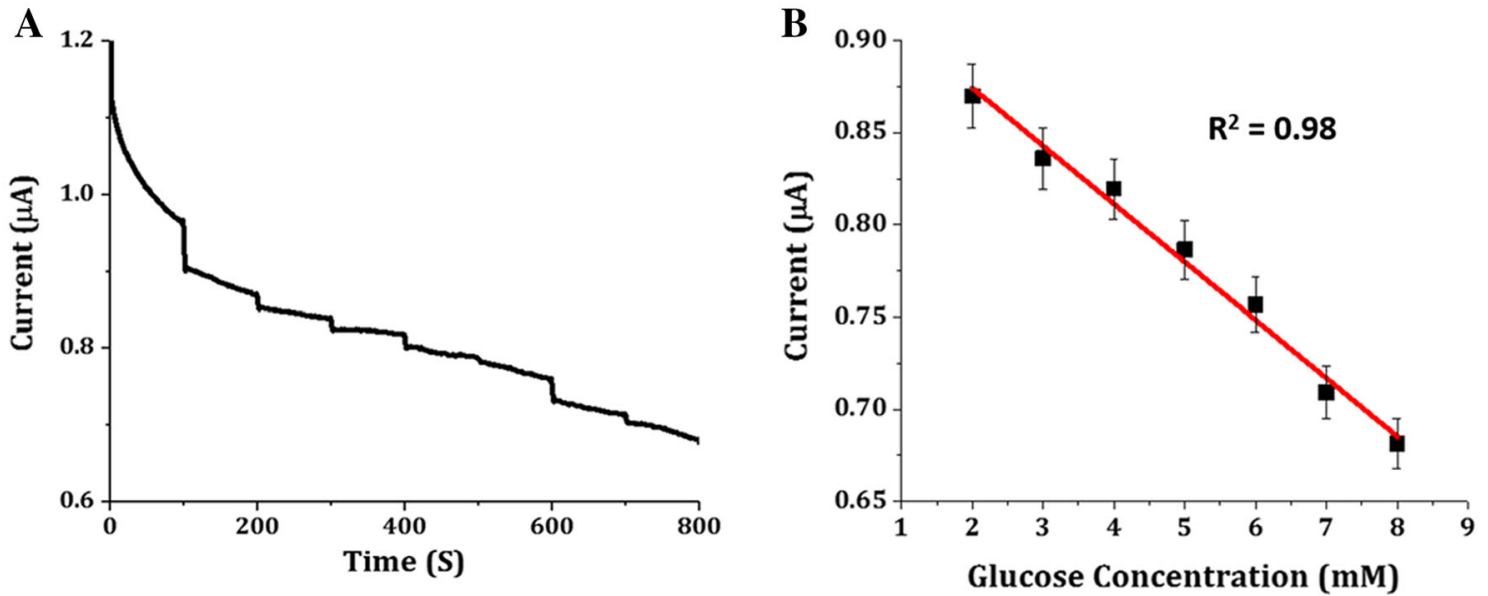

Fig. 6 a Amperometric pattern of CNT/GCE for different concentrations of glucose and b plot of concentration of glucose versus current showing the linear range of the developed biosensor 
Table 2 Comparison of the developed glucose biosensor with previous reports

\begin{tabular}{llll}
\hline Interface & Detection range $(\mathrm{mM})$ & Real-time sample & Reference \\
\hline $\mathrm{CuO}$ flowers & $0.004-8$ & - & Wang et al. (2010) \\
$\mathrm{ZnO}$ flowers & $0.01-10$ & - & Ibupoto et al. (2013) \\
$\mathrm{Pt}-\mathrm{Pb}$ & $0-16$ & - & Wang et al. (2008a) \\
$\mathrm{Ni}(\mathrm{OH})_{2}-\mathrm{NND}$ & $1-9$ & - & Ko et al. (2013) \\
$\mathrm{Pt}$ flowers on SWCNT & $0.002-10$ & - & Su et al. (2010) \\
Mesoporous platinum & $0-10$ & - & Park et al. (2006) \\
MWCNTs & $2-11$ & - & Ye et al. (2004) \\
MWCNTs & - & Macrophage-cultured medium & Wang et al. (2007) \\
MWCNTs & $2-8$ & & Present work \\
\hline
\end{tabular}

the addition of glucose indicating its specificity at the given potential.

The detection of glucose in samples has wide-range applications in many fields that include health, food, beverages, and biotechnology. The use of enzyme-based sensors becomes expensive due to their non-reusable nature. The use of enzymeless sensor with a better stability and reusability without compromising the selectivity can overcome the limitations of enzyme-based sensors. The CNT-coated GCE reported in the present study exhibits high selectivity and rapid response. The sensor could be reused for 20 times with a decrease of only $11.9 \%$ in current recorded after 20 runs. The dry stability of the sensor was evaluated at room temperature and it was found that after 7 days, a $22.6 \%$ decrease in the peak current.

\section{Measurement of glucose in macrophage-cultured cell culture medium}

To evaluate the utility of the developed sensor for measuring glucose in biological samples, the sensor was employed to determine the glucose utilization of macrophages in the unstimulated as well as stimulated states. Spent medium collected from the culture plates was used for amperometric measurements. Amperometry was performed by applying a constant potential of $-0.32 \mathrm{~V}$ over a period of $100 \mathrm{~s}$. The measured current is converted to glucose value using the standard plot constructed using known quantities of glucose. Measured glucose values were subtracted from supplemented glucose concentrations, so that glucose utilization was calculated. Glucose utilization in unstimulated and stimulated macrophages is depicted in Fig. 8.

It is observed that macrophages in the unstimulated condition showed a slight but progressive increase in the glucose utilization values with increasing cell numbers. However, the increase was only $5 \%$ at a seeding density of 10,000 cells when compared to the glucose utilized by

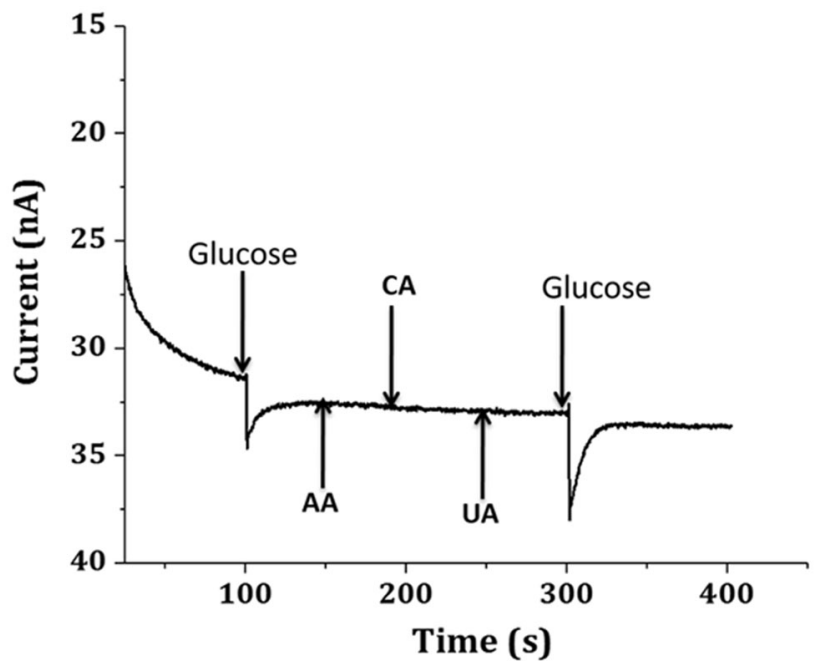

Fig. 7 Amperometric detection of glucose and other interferents in PBS at an applied potential of $-0.32 \mathrm{~V}$

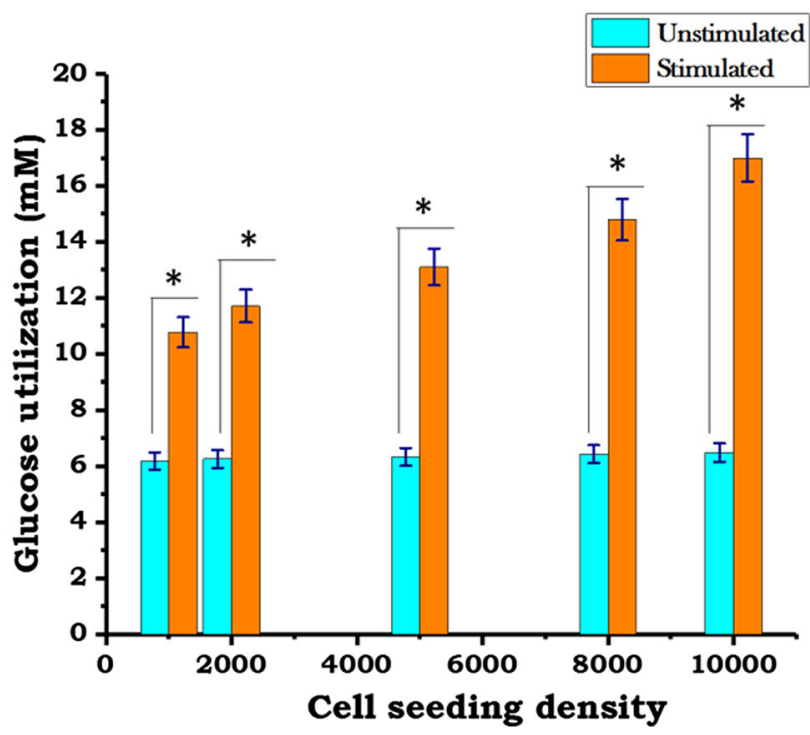

Fig. 8 Glucose utilization in stimulated and unstimulated macrophages with respect to the cell seeding densities 

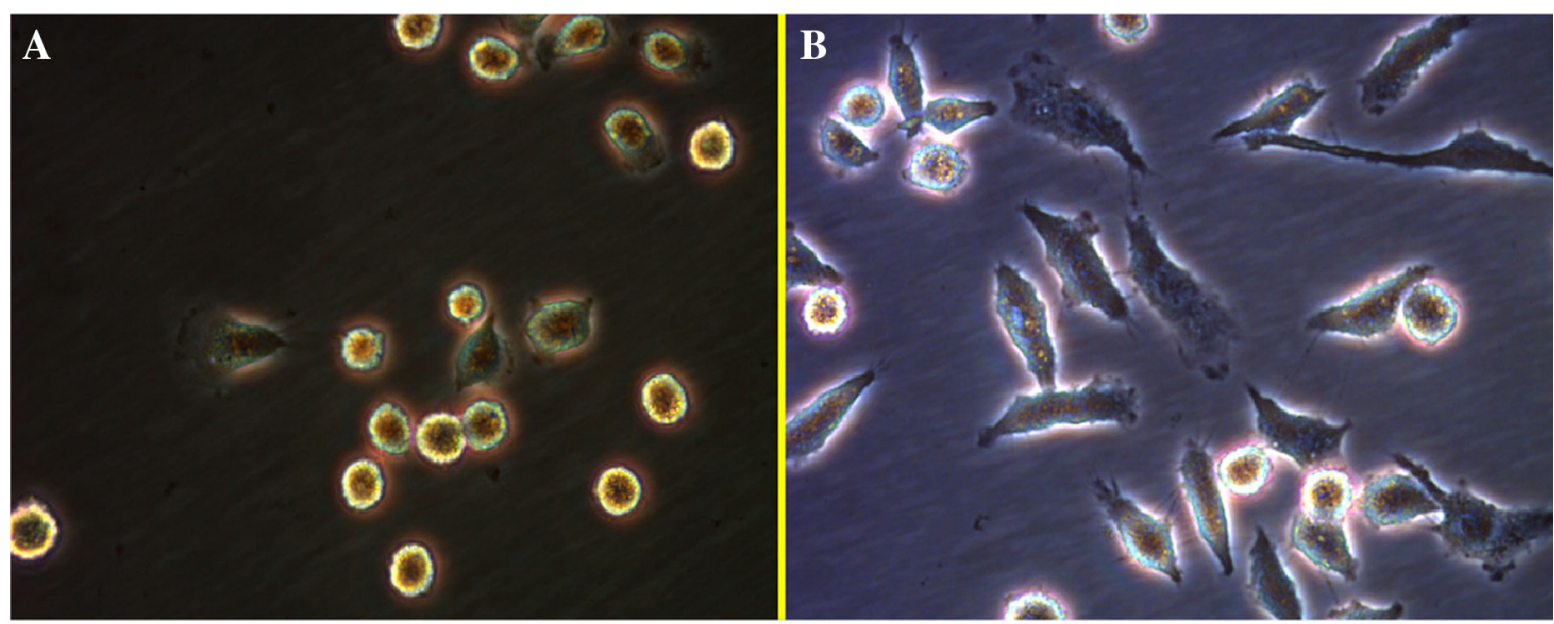

Fig. 9 Inverted microscope images of unstimulated (a) and stimulated (b) macrophages $(\times 40$ magnification)

1000 cells. This suggests that the cells are in the quiescent phase. In contrast, the cells stimulated with LPS showed a significantly higher glucose utilization at all seeding densities when compared with their unstimulated counterparts. A distinct increase in the glucose utilization was observed with increasing cell numbers in the LPS-stimulated macrophages. A $67 \%$ increase in glucose utilization was measured in the stimulated macrophages at a seeding density of 10,000 when compared with the values obtained for 1000 cells. A 1.6- to 2.6-fold increase in glucose utilization was observed in the LPS-stimulated macrophages when compared with the unstimulated cells. This difference in glucose utilization between the stimulated and unstimulated macrophages arises due to the active remodeling of the actin cytoskeletal network that occurs under stimulated conditions leading to a pronounced morphological transformation in the macrophage (Fig. 9). A recent report has demonstrated that LPS-stimulated macrophages exhibit down-regulation of ATP synthesis from fatty acid oxidation and hence is heavily dependent on the glycolytic pathway and not the oxidative phosphorylation to meet the energy demands associated with the cytoskeletal remodeling (Venter et al. 2014). Our data support this mechanism as we observe a distinct twofold increase in the glucose utilization by macrophages stimulated with LPS.

\section{Concluding remarks}

Carbon nanotubes have been widely explored as an interface material along with an enzyme for electrochemical detection of various analytes including glucose. The present work demonstrates the intrinsic catalytic ability of MWCNTs. Along with their excellent electron transfer capability, the MWCNTs formed a catalytic interface for efficient electrochemical enzymeless detection of glucose within $3 \mathrm{~s}$. The biosensor exhibited a linear range from 2 to $8 \mathrm{mM}$ with a limit of detection $1.59 \mathrm{mM}$ that is superior to many enzymeless glucose sensors reported in literature. The developed biosensor is unaffected by the potential interferents. These attributes enable real-time detection of glucose with the developed biosensor that may find applications in clinical diagnostics. The sensor was found to effectively detect glucose utilization in stimulated and unstimulated macrophages that can be explored in future for clinical diagnosis of inflammatory disorders.

Acknowledgements Sasya Madhurantakam wishes to acknowledge Department of Science and Technology for providing INSPIRE fellowship (DST/IF/120812). The authors are gratefully thank Department of Science and Technology, Government of India, for providing cell culture facility through DST-FIST (SR/FST/LSI-453/2010). Authors are pleased to acknowledge SASTRA University for providing financial and infrastructural support to carry out this research.

Open Access This article is distributed under the terms of the Creative Commons Attribution 4.0 International License (http:// creativecommons.org/licenses/by/4.0/), which permits unrestricted use, distribution, and reproduction in any medium, provided you give appropriate credit to the original author(s) and the source, provide a link to the Creative Commons license, and indicate if changes were made.

\section{References}

Asefa T, Duncan CT, Sharma KK (2009) Recent advances in nanostructured chemosensors and biosensors. The Analyst 134:1980-1990. doi:10.1039/b911965p

Bokobza L, Zhang J (2012) Raman spectroscopic characterization of multiwall carbon nanotubes and of composites. Express Polym Lett 6:601-608. doi:10.3144/expresspolymlett.2012.63

Cao Z, Zou Y, Xiang C et al (2007) Amperometric glucose biosensor based on ultrafine platinum nanoparticles. Anal Lett 40:2116-2127. doi:10.1080/00032710701566909

Chen J, Zhang W-D, Ye J-S (2008) Nonenzymatic electrochemical glucose sensor based on $\mathrm{MnO}_{2} / \mathrm{MWNTs}$ nanocomposite. Electrochem Commun 10:1268-1271. doi:10.1016/j.elecom.2008.06. 022 
Chen J, Zheng X, Miao F (2012) Engineering graphene/carbon nanotube hybrid for direct electron transfer of glucose oxidase and glucose biosensor. J Appl Electrochem 42:875-881. doi:10. 1007/s10800-012-0461-x

Dantas LMF, Castro PS, Peña RC, Bertotti M (2014) Amperometric determination of hydrogen peroxide using a copper microelectrode. Anal Methods 6:2112. doi:10.1039/c3ay41980k

Dong S, Chen X (2002) Some new aspects in biosensors. Rev Mol Biotechnol 82:303-323. doi:10.1016/S1389-0352(01)00048-4

Freemerman AJ, Johnson AR, Sacks GN et al (2014) Metabolic reprogramming of macrophages: glucose transporter 1 (GLUT1)mediated glucose metabolism drives a proinflammatory phenotype. J Biol Chem 289:7884-7896. doi:10.1074/jbc.M113.522037

Harper A, Anderson MR (2010) Electrochemical glucose sensorsdevelopments using electrostatic assembly and carbon nanotubes for biosensor construction. Sensors 10:8248-8274. doi:10.3390/ s100908248

Hu S, Hu C (2009) Carbon nanotube-based electrochemical sensors: principles and applications in biomedical systems. J Sens. doi: $10.1155 / 2009 / 187615$

Ibupoto Z, Khun K, Beni V, Willander M (2013) Non-enzymatic glucose sensor based on the novel flower like morphology of nickel oxide. Soft Nanosci Lett 3:46-50. doi:10.4236/snl.2013.34A013

Kimmel DW, LeBlanc G, Meschievitz ME, Cliffel David E (2013) Electrochemical sensors and biosensors. Anal Chem 84:685-707. doi:10.1021/ac202878q.Electrochemical

Ko C-Y, Huang J-H, Raina S, Kang WP (2013) A high performance non-enzymatic glucose sensor based on nickel hydroxide modified nitrogen-incorporated nanodiamonds. The Analyst 138:3201-3208. doi:10.1039/c3an36679k

Lei Y, Yan X, Luo N et al (2010) ZnO nanotetrapod network as the adsorption layer for the improvement of glucose detection via multiterminal electron-exchange. Colloids Surf A 361:169-173. doi:10.1016/j.colsurfa.2010.03.029

Li Z, Chen Y, Xin Y, Zhang Z (2015) Sensitive electrochemical nonenzymatic glucose sensing based on anodized $\mathrm{CuO}$ nanowires on three-dimensional porous copper foam. Sci Rep 5:16115. doi:10.1038/srep16115

Lo PH, Kumar SA, Chen SM (2008) Amperometric determination of $\mathrm{H}_{2} \mathrm{O}_{2}$ at nano- $\mathrm{TiO}_{2} / \mathrm{DNA} /$ thionin nanocomposite modified electrode. Colloids Surf B 66:266-273. doi:10.1016/j.colsurfb.2008. 07.003

Ma Y, Di J, Yan X et al (2009) Direct electrodeposition of gold nanoparticles on indium tin oxide surface and its application. Biosens Bioelectron 24:1480-1483. doi:10.1016/j.bios.2008.10. 007

Maltby S, Khazaie K, McNagny KM et al (2009) Mast cells in tumor growth: angiogenesis, tissue remodelling and immune-modulation. Biochim Biophys Acta 1796:19-26. doi:10.1146/annurev. immunol.021908.132620.Immune

Niu XH, Shi LB, Zhao HL, Lan MB (2016) Advanced strategies for improving the analytical performance of Pt-based nonenzymatic electrochemical glucose sensors: a minireview. Anal Methods 8:1755-1764. doi:10.1039/C5AY03181H

Numata T, Takahashi K, Inoue R (2016) "TRP inflammation" relationship in cardiovascular system. Semin Immunopathol 38:339-356. doi:10.1007/s00281-015-0536-y

Park S, Boo H, Chung TD (2006) Electrochemical non-enzymatic glucose sensors. Anal Chim Acta 556:46-57. doi:10.1016/j.aca. 2005.05.080

Reitz E, Jia W, Gentile M et al (2008) CuO nanospheres based nonenzymatic glucose sensor. Electroanalysis 20:2482-2486. doi:10.1002/elan.200804327

Salimi A, Compton RG, Hallaj R (2004) Glucose biosensor prepared by glucose oxidase encapsulated sol-gel and carbon-nanotube- modified basal plane pyrolytic graphite electrode. Anal Biochem 333:49-56. doi:10.1016/j.ab.2004.06.039

Sattayasamitsathit S, Thavarungkul P, Thammakhet C et al (2009) Fabrication of nanoporous copper film for electrochemical detection of glucose. Electroanalysis 21:2371-2377. doi:10. 1002/elan.200904697

Su L, Jia W, Zhang L et al (2010) Facile synthesis of a platinum nanoflower monolayer on a single-walled carbon nanotube membrane and its application in glucose detection. J Phys Chem C 114:18121-18125. doi:10.1021/jp107636r

Sue J-W, Hung C-J, Chen W-C, Zen J-M (2008) Amperometric determination of sugars at activated barrel plating nickel electrodes. Electroanalysis 20:1647-1654. doi:10.1002/elan.200804228

Suneesh PV, Chandhini K, Ramachandran T et al (2013) Tantalum oxide honeycomb architectures for the development of a nonenzymatic glucose sensor with wide detection range. Biosens Bioelectron 50:472-477. doi:10.1016/j.bios.2013.07.007

Thiagarajan V, Madhurantakam S, Sethuraman S et al (2016) Nano interfaced biosensor for detection of choline in triple negative breast cancer cells. J Colloid Interface Sci 462:334-340. doi:10. 1016/j.jcis.2015.10.014

Venter G, Oerlemans FTJJ, Wijers M et al (2014) Glucose controls morphodynamics of LPS-stimulated macrophages. PLoS ONE. doi:10.1371/journal.pone.0096786

Wang J, Sun X, Cai X et al (2007) Nonenzymatic glucose sensor using freestanding single-wall carbon nanotube films. Electrochem Solid-State Lett 10:J58-J60. doi:10.1149/1.2710184

Wang J, Thomas DF, Chen A (2008a) Based on nanoporous PtPb networks glucose sensor based on three-dimensional $\mathrm{PtPb}$ netone-step hydrothermal method. The surface morphology. Anal Chem 80:997-1004. doi:10.1021/ac701790z

Wang Y, Xu H, Zhang J, Li G (2008b) Electrochemical sensors for clinic analysis. Sensors 8:2043-2081. doi:10.3390/s8042043

Wang X, Hu C, Liu H et al (2010) Synthesis of $\mathrm{CuO}$ nanostructures and their application for nonenzymatic glucose sensing. Sens Actuators B 144:220-225. doi:10.1016/j.snb.2009.09.067

Wang Y, Liu L, Li M et al (2011) Multifunctional carbon nanotubes for direct electrochemistry of glucose oxidase and glucose bioassay. Biosens Bioelectron 30:107-111. doi:10.1016/j.bios. 2011.08.038

Wang G, He X, Wang L et al (2013) Non-enzymatic electrochemical sensing of glucose. Microchim Acta 180:161-186. doi:10.1007/ s00604-012-0923-1

Ye J-S, Wen Y, De Zhang W et al (2004) Nonenzymatic glucose detection using multi-walled carbon nanotube electrodes. Electrochem Commun 6:66-70. doi:10.1016/j.elecom.2003.10.013

Yuan J, Wang K, Xia X (2005) Highly ordered platinum-nanotubule arrays for amperometric glucose sensing. Adv Func Mater 15:803-809. doi:10.1002/adfm.200400321

Zhang X, Liao Q, Chu M et al (2014) Structure effect on graphenemodified enzyme electrode glucose sensors. Biosens Bioelectron 52:281-287. doi:10.1016/j.bios.2013.07.022

Zhu J, Chen X, Yang W (2010) A high performance electrochemical sensor for NADH based on graphite nanosheet modified electrode. Sens Actuators B 150:564-568. doi:10.1016/j.snb.2010.08.039

Zhu Z, Garcia-Gancedo L, Flewitt AJ et al (2012) A critical review of glucose biosensors based on carbon nanomaterials: carbon nanotubes and graphene. Sensors 12:5996-6022. doi:10.3390/ s120505996

\section{Publisher's Note}

Springer Nature remains neutral with regard to jurisdictional claims in published maps and institutional affiliations. 